\title{
The role of gut microbiome in obesity: a systematic review
}

\author{
E. Alamri ${ }^{1}$, H. Bayomy ${ }^{1}$ and Z. Mohammedsaledh ${ }^{2}$ \\ ${ }^{1}$ Nutrition and food science department, University of Tabuk, Saudi Arabia and \\ ${ }^{2}$ Medical laboratory department, University of Tabuk, Saudi Arabia
}

Obesity is worldwide epidemic given its rapid growth in global prevalence ${ }^{(1)}$. Among the risk factors contributing to obesity, human gut microbiome recently emerges as a new factor ${ }^{(2)}$. The aim of the present review is to evaluates the role of the gut microbiome in obesity.

We used the methods recommended by the Centre for Reviews and Dissemination, University of York ${ }^{(3)}$.and followed the Preferred Reporting Items for Systematic Reviews and Meta-Analyses (PRISMA) statement ${ }^{(4)}$. Several databases were used for searching (PubMed, Science Direct, Web of Science, and Google Scholar). Observational human studies and clinical trials that evaluated the gut microbiota composition in adults with obesity and in those of normal weight by any validated technique, written in English were included. Studies with animal models, children, teenagers and pregnant; clinical trials with no gut microbiota composition baseline data; evaluation of oral, stomach, skin or oropharyngeal microbiota; studies not compared with lean individuals; and studies with a high risk of bias (poor quality), were excluded. Quality assessment and risk of bias was assessed using a standardized form consisting of 22 criteria, including information about study setting, population and design, sample selection, baseline characteristics. Studies which conducted between January 2010 and January 2021 were included. Literature searches were performed using the keywords, gut microbes, gut microbiome, obesity, and 'weight gain. The initial search yielded 150 articles and only 15 articles met the criteria to be included in this review.

SIX studies examined the microbiota of obese human subjects' pre- and post-weight loss and reported; loss of body weight correlated with a decrease in Firmicutes and an increase in Bacteroidetes, regardless of whether patients maintained a fat-restrictive or carbohydrate-restrictive diet. Five studies reported elevated levels of Actinobacteria, lower levels of Bacteroidetes, and lower microbial diversity in obese versus lean subjects, but no significant difference in abundance of Firmicutes, while four studies found that obese subjects had much higher levels of Bacteroidetes and lower Firmicutes than their lean counterparts.

In conclusion, the role of the gut microbiota as a contributor to obesity is complex. It should be noted that this diseases have multiple contributing factors and underlying etiologies. The possibilities of microbial effects are simply one piece of a complex puzzle, and that piece may vary in size depending on the individual studied and the environment to which he or she is accustomed.

\section{References}

1. World Health Organization (2016). [Available at: https://www.who.int/news-room/fact-sheets/detail/obesity-and-overweight].

2. Kunnackal JG \& Mullin GE (2016) Current oncology reports, 2016, 18.7, 1-7.

3. Centre for Reviews and Dissemination. (2009) York: Centre for Reviews and Dissemination, University of York.

4. Moher D, Liberati A, Tetzlaff J et al. (2009) Ann Intern Med. 151(4), 264-9. 